EPJ Web of Conferences 110,01039 (2016)

DOI: $10.1051 /$ epjconf/201611001039

(C) Owned by the authors, published by EDP Sciences, 2016

\title{
HIGH-TEMPERATURE HEATING OF LIQUID IN A ROUND TUBE IN TERMS OF AXIAL HEAT CONDUCTION
}

G. Medvedev ${ }^{1, a}$

${ }^{1}$ National Research Tomsk Polytechnic University, 634050 Tomsk, Russia

\begin{abstract}
The given paper presents the calculation method of high-temperature liquid heating in channels under the nonlinear boundary conditions and studies the influence of axial thermal conduction on temperature distribution.
\end{abstract}

\section{Introduction}

It is commonly assumed in tube heat transfer problems that density variation of heat flux conditioned by thermal conduction along an axis is small in comparison with radial variation. In other words thermal conduction is neglected by reason of axial thermal conduction [2, 3]. In most cases this assumption puts to the test well. However it can lead to significant errors if the Peclet's number $\mathrm{Pe}>>100$ and $\mathrm{X} / 2 r_{0} \geq 1 \quad$ [1]. For gases $\left(P_{r} \approx 1\right)$ and nonmetallic liquids $\left(P_{r} \approx 1 \div 1000\right)$ this assumption is mostly realized. But for liquid metals $\left(P_{r} \approx 0,005 \div 0,05\right)$ it may not be realized. In that respect in energy equation the member $\partial^{2} \theta(X, R) / \partial X^{2}$ must be retained, because it takes into account heat flux variation due to conduction along an axis.

\section{Problem statement}

In a channel of circular section the liquid undergoes (fig. 1). There is a laminar and developed liquid flow. The outside tube walls are washed by high-temperature gas filling. The heat transfer on the outer surface keeps in both with the Newton's and StefanBoltzmann's laws.

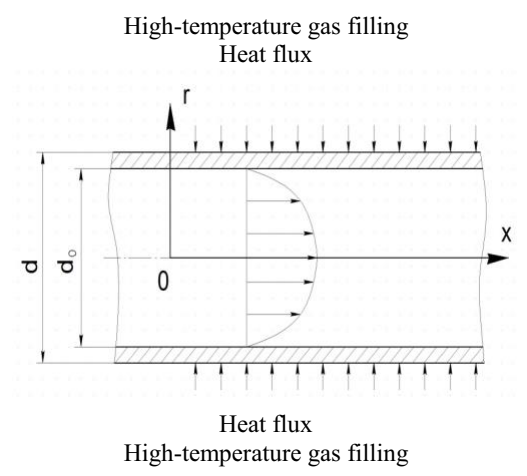

Figure 1. Physical analogue and coordinate system.

In that case the problem resolves itself to the solution of energy equation (1) with boundary conditions (2) - (4) written as

$$
\frac{\partial^{2} \theta(X, R)}{\partial R^{2}}+\frac{1}{R} \frac{\partial \theta(X, R)}{\partial R}+\frac{1}{P e^{2}} \frac{\partial^{2} \theta(X, R)}{\partial X^{2}}=\left(1-R^{2}\right) \frac{\partial \theta(X, R)}{\partial X}
$$

\footnotetext{
${ }^{\mathrm{a}}$ Corresponding author: ggm@tpu.ru
} 


\section{EPJ Web of Conferences}

$$
\begin{gathered}
X / 2 r_{0} \geq 1 \\
\frac{\partial \theta(X, 0)}{\partial R}=0 \\
\frac{\partial \theta(x, 1)}{\partial R}=B_{i}[1-\theta(x, 1)]+\operatorname{Sk}\left[1-\theta^{4}(x, 1)\right]
\end{gathered}
$$

Here

$$
\begin{aligned}
& \theta_{0} \leq \theta=\frac{T}{T_{c}} \leq 1, \\
& 0 \leq x=\frac{2}{P e} \frac{x}{d_{0}}<\infty, \\
& B_{i}=\frac{\alpha}{\lambda} r_{0} \frac{d}{d_{0}},
\end{aligned}
$$$$
\begin{aligned}
& 0 \leq R=\frac{r}{r_{0}} \leq 1, \\
& P e=\frac{\varpi}{a} d_{0},
\end{aligned}
$$$$
S k=\frac{T_{c}^{3} r_{0} \sigma}{\lambda} \frac{d}{d_{0}}
$$

The original equation (1) depends not only on coordinates $\mathrm{X}$ and $\mathrm{R}$ but also on the Peclet's number which occurs together with the member taking into account conduction along an axis.

It is possible to avoid difficulties emerging in connection with nonlinear boundary condition which reflects convection-andradiation heat transfer on the tube surface with the help of transition to the system with linear boundary conditions. For that reason we use a new variable $\mathrm{U}(\mathrm{X}, \mathrm{R})$ which is expressed by the relation [4]:

To make the calculations easier in [3] the graphic calculations of transcendental equation (5) at different values $\mathrm{Bi} / \mathrm{S}$ are given.

The transformation function (5) without changes of symmetry conditions (3)

$$
\frac{d U(X, 0)}{d R}=0,
$$

linearizes the boundary condition (4) resulting in third class linear boundary condition

$$
\frac{d U(X, 0)}{d R}=-p \operatorname{Sk} U(X, 1)
$$

the temperature condition at the channel inlet will be written as

$$
U(0, R)=\exp -p \int_{0}^{\theta} \frac{d \theta(X, R)}{B i / S k[1-\theta(X, R)]+1-\theta^{4}(X, R)}=U_{0}
$$

and energy equation (1) for a new variable $\mathrm{U}(\mathrm{X}, \mathrm{R})$ will be as follows

$$
\frac{d^{2} U(X, R)}{d R^{2}}+\frac{1}{R} \frac{d U(X, R)}{d R}+\frac{1}{P e^{2}} \frac{\partial^{2} U(X, R)}{\partial X^{2}}=\left(1-R^{2}\right) \frac{d U(X, R)}{d X}+F(X, R),
$$

where

$$
F(X, R)=\frac{p U}{\left[\frac{B i}{S k}(1-\theta)+1-\theta^{4}\right]^{2}}\left[\left(\frac{\partial \theta}{\partial R}\right)^{2}+\left(\frac{1}{P e} \frac{\partial \theta}{\partial X}\right)^{2}\right] *\left(p-\frac{B i}{S k}-4 \theta^{3}\right)
$$

The modified equation system (6) - (9) keeps all nonlinear characteristics of the original problem (1) - (4).

To find the problem (6) - (9) solution it is necessary to minimize the nonlinear function (10) in advance, which in accordance with physical meaning is represented as autothermal heat supply (heat sink) of variable intensity. This manipulation is performed with the help of adjustment factor $p$ containing in the transformation function (5).

It stands to mathematical and physical reasons that introduction of the adjustment factor $\mathrm{p}$ to linearizing transformation (5) allows to minimize the function (10). It is true that if $p \rightarrow B i / S k+4 \theta^{3}$, then $F(X, R) \rightarrow 0$. 


\section{Thermophysical Basis of Energy Technologies 2015}

If the temperature differential on the pipe section is not big, then to receive the condition $F(X, R)=0$ it is sufficient to suppose that

$$
p=\frac{B i}{S k}+4 \theta^{3}
$$

where $\theta$ is determined as $\theta=\frac{1}{2}\left(\theta_{0}+1\right)$

It allows us for determination $\theta=\theta(X, R)$ to use the problem solution at $F(X, R)=0$ [5].

$$
F(X, R)=U_{0} \sum_{n 0} C_{n} \varphi_{n}(R) \exp \left(-\beta_{n}^{2} X\right)
$$

and transformation

$$
Z=\frac{\ln U(X, R)}{-p}=\int_{0}^{\theta} \frac{d \theta(X, R)}{\left[\frac{B i}{S k}[(1-\theta(X, R))]+1-\theta^{4}(X, R)\right]}
$$

The values of constants $C_{n}, \beta_{n}$ and fundamental function $\varphi_{m}=\varphi_{m}(R)$ are given in [5].

For the purpose of defining the influence of axial thermal conduction on temperature distribution on the basis of selected model the calculation of temperatures on the tube surface at such values: $P e \rightarrow \infty ; P e=1.0 ; P e=10$; was performed; relation $\frac{B i}{S k}=1.0$ and temperature at the heated inlet is equal to $\theta_{2}=0.2$ (fig. 2).

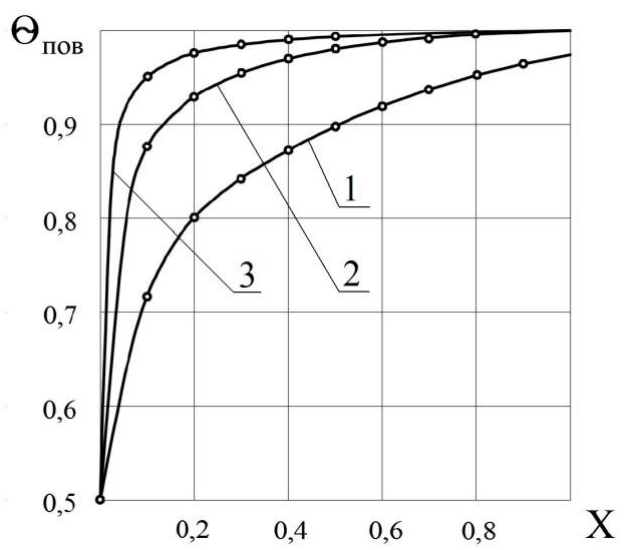

Figure 2. Influence of axial thermal conduction on surface temperatures values $(1-P e=1,0 ; 2-P e=10 ; 3$ - without considering axial thermal conduction).

As can be seen from Fig. 2 the influence of axial thermal conduction the larger is, the less the Pe number is.

With the rising of criteria $\mathrm{Sk}, \mathrm{Bi}$ and decrease of $\theta_{0}$ the pipe section temperature differential increases in accordance with the value of nonlinear function (10). As a consequence while calculating intensive convection-and-radiation heat it is necessary to divide all the temperature range $\theta$ (from 0 to 1.0 ) into two or more sections (in general into $\mathrm{N}$ ) and for each i-section factor $\mathrm{p}$ according to the formula:

$$
\begin{gathered}
p_{i}=\frac{B i}{S k}+4 \theta_{i}^{3}, i=1,2,3 \ldots . . N-1 \\
p_{N}=\frac{B i}{S k}+4\left(\frac{\theta_{N-1}+1}{2}\right)^{3}
\end{gathered}
$$

If the number of intervals is greater than unity, then temperature distribution in liquid flow found for any section is used later as the initial condition fro the next section. 


\section{EPJ Web of Conferences}

\section{Conclusion}

On the basis of approximate approach to linearizing functions the problem of complex heat transfer laminar flow in the round tube was solved. It is established that axial heat channel influences temperature distribution on the tube surface.

\section{References}

1. B.S. Petukhov. Teploobmen i soprotivlenie pri laminarnom techenii zhidkosti v trubakh (Energiya, 1967)

2. V.V. Ivanov, G.G. Medvedev. Raspredelenie temperatur v laminarnom potoke zhidkosti pri luchisto-konvektivnom nagreve. Izvestiya AN SSSZ (Energetika i transport, № 3, 1970)

3. Y.V. Vidin, V.V. Ivanov, G.G. Medvedev. Raschet teploobmena pri laminarnom techenii zhidkosti v kanalakh (Izdatel'stvo Krasnoyarskogo politekhnicheskogo instituta, 1971)

4. V.V. Ivanov. Phizika i khimiya obrabotki materialov, (5), (1972)

5. Hsu Chia-Jung, Chemical Engineering Science, 23, (1968) 